\title{
Preparation of a composite fibrous membrane loaded with mesalazine and metronidazole by interlaced electrospinning
}

\author{
ZHENRAN WANG and JIAN SUO \\ Department of General Surgery, The First Hospital of Jilin University, Changchun 130021, P.R. China
}

Received August 30, 2011; Accepted November 7, 2011

DOI: $10.3892 / \mathrm{mmr} .2011 .670$

\begin{abstract}
Novel composite fibrous membranes loaded with mesalazine and metronidazole were fabricated via interlaced electrospinning. The fibers were characterized by scanning electron microscopy and FTIR transmission spectra techniques. These characterizations were performed in the aim of optimizing the experimental conditions which allowed us to obtain good morphology of fibrous membrane loaded drugs. The in vitro release experiments revealed that mesalazine and metronidazole were released continuously from the loaded drug fibrous membrane. The fibrous membrane-loaded drugs also showed excellent stability. Compared to those of other drug delivery systems, the main advantage of these two fibrous membrane-loaded drugs is that they can be directly implanted as a lesion after surgery to inhibit recurrence in Crohn's disease.
\end{abstract}

\section{Introduction}

Crohn's disease (CD) is an inflammatory bowel disease and its cause is obscure $(1,2)$. Since the symptoms of CD are similar to those of other intestinal disorders, such as irritable bowel syndrome and ulcerative colitis, it can be difficult to diagnose. Ulcerative colitis causes inflammation and ulcers in the top layer of the lining of the large intestine. In CD, all layers of the intestine may be involved, and normal healthy bowel can be found between sections of diseased bowel.

$\mathrm{CD}$ is an ongoing disorder that causes inflammation of the digestive tract, also referred to as the gastrointestinal (GI) tract. CD may affect any area of the GI tract, from the mouth to the anus, but it most commonly affects the lower part of the small intestine, called the ileum. The swelling extends deep into the lining of the affected organ. The swelling causes pain and makes the intestines empty frequently, resulting in diarrhea.

Treatment may include drugs, nutritional supplements, surgery or a combination of these options. The goals of treatment are to control inflammation, correct nutritional

Correspondence to: Dr Jian Suo, Department of General Surgery, The First Hospital of Jilin University, No. 71 Xin Min Street, Changchun 130021, P.R. China

E-mail: suojianwcwk@163.com

Key words: interlaced electrospinning, mesalazine, metronidazole deficiencies, and relieve symptoms such as abdominal pain, diarrhea and rectal bleeding. At present, treatment helps control the disease by lowering the number of times a person experiences a recurrence, but there is no cure. Treatment for $\mathrm{CD}$ depends on the location and severity of the disease, complications and the response of an individual to previous medical treatments when treated for recurring symptoms (3).

Chemically, mesalazine (MEZ) is a 5-amino salicylic acid (4). It is an anti-inflammatory drug structurally related to salicylates, which is active in inflammatory bowel disease (5). Fig. 1A shows its molecular structure. MEZ diminishes inflammation by inhibiting cyclooxygenase and lipoxygenase, thereby decreasing the production of prostaglandins, leukotrienes (CTB4) and hydroxyeicosatetraenoic acids (HETEs), respectively. It appears to be topical rather than systemic. Common oral administration facilitates absorption of MEZ in the small intestine, reduces the time of drug arrival in the colon and causes side effects, such as diarrhea, nausea, cramping, flatulence and headache (6). The recurrence of ulcerative colitis is related to re-breeding infection of clostridium bacteria.

Metronidazole (7) is an amebicide antiprotozoal and antibiotic effective against anaerobic bacteria and certain parasites. Its molecular structure is shown in Fig. 1B. It is the drug of choice for first episodes of mild-to-moderate Clostridium difficile infection (8). Metronidazole kills Bacteroides fragilis and Clostridium perfringens more rapidly than clindamycin $(9,10)$. It also involves the immune system, hampering adhesion between leucocytes and endothelial cells, and inhibiting immigration of inflammatory cells (11). The combination of MEZ and metronidazole alleviates the suffering of the patient during active episodes of ulcerative colitis.

Prevention of post-operative recurrences in $\mathrm{CD}$ is a complex problem. In the past, the strategy was to administer no treatment until clinical recurrence. An alternative approach is to administer no treatment, and then to treat the patient according to the severity of the endoscopic recurrence.

Electrospinning is a useful and relatively simple method for producing fibers with a submicrometer diameter range. Using this method, it is possible to obtain ultra-fibers from various types of polymers, such as polyolefins (12), polyamides (13), polyesters (14), polyurethanes (15), polypeptides and DNA $(16,17)$ as well as polymers with special properties (18). In recent years, probably more than 100 different polymers, copolymers and mixtures of polymers have been successfully used to obtain fibers with diameters of less than $1 \mu \mathrm{m}$. Since 
MEZ and metronidazole have difficulty dissolving in the same solvent, we developed a new fibrous membrane by interlaced electrospinning, with concurrent loading of the above two drugs. The amount of loaded drugs may be adjusted to a suitable range. These two fibrous membrane-loaded drugs can then be directly implanted as a lesion after surgery, improving the therapeutic index and reducing drug dosage, toxicity and side effects.

\section{Materials and methods}

Materials. PLGA (Mn 70,000; LA:GA 50:50) was purchased from the Daigang Biomaterials Co., Ltd. (Jinan, China). MEZ and metronidazole were purchased from the Laifu Chemical Drug Co., Ltd. (Zhousan, China) and the Yancheng Pharmacy (Yancheng, China), respectively. Chloroform, dimethylfomamide and ethanol were of analytical grade and used without further purification. Methanol was of chromatographic grade. Tetrabutylammonium bromide was obtained commercially.

Preparation of the fibrous membrane-loaded drugs. Preparation of the different solutions was as follows. PLGA was dissolved in chloroform to prepare a $10 \%$ wt solution. Incorporation of the metronidazole ( $2 \%$ by weight of the polymer) was carried out by dissolving the drug directly in the as-prepared polymer solution. A suitable amount of MEZ was dissolved in DMF, and then chloroform was added (chloroform/DMF, $3 / 2$ weight ratio). PLGA was dissolved in the mixed solvent system to prepare a $10 \%$ wt solution.

An interlaced electrospinning setup schematic diagram is shown in Fig. 1. Electrospinning was carried out using a variable high-voltage power supply, which produces voltages ranging from 0 to $10 \mathrm{kV}$. It consists of two positive-power supplies attached to a blunt steel needle and one negativepower supply attached to the counter electrode. The syringes containing the different solution were attached to the blunt needle and were put into a syringe pump ejecting $50 \mu \mathrm{l}$ of the solutions per min through the blunt needle. A stainless steel drum $(\mathrm{d}=15 \mathrm{~cm})$ which may slowly rotate served as the counter electrode. It was covered with aluminum foil for the collection of the different fibers.

Characterization of the fibrous membrane-loaded drugs. Morphological appearances of ultra-fine mats were observed on a scanning electron microscope (SEM; Hidachi S-2350) after gold coating. Fourier transform infrared (FT-IR) spectra of celluloses were obtained with a Nicolet Magna-IR 560 spectrophotometer.

In vitro drug release. Preparation of phosphate-buffered saline (PBS) was as follows. The phosphate buffer solution was prepared by mixing $0.2 \mathrm{M}$ monobasic potassium phosphate $\left(\mathrm{KH}_{2} \mathrm{PO}_{4}\right)$ aqueous solution with $0.2 \mathrm{M}$ sodium hydroxide $(\mathrm{NaOH})$ aqueous solution to attain a $\mathrm{pH}$ of 6.8 .

A piece of the fibrous membrane loaded with the drugs $\left(\sim 2 \times 2 \mathrm{~cm}^{2}\right)$ was placed in $40 \mathrm{ml}$ of $0.05 \mathrm{M}, \mathrm{pH} 6.8 \mathrm{PBS}$. The test was performed in a $37^{\circ} \mathrm{C}$ incubator-shaker at $50 \mathrm{rpm}$. At appropriate intervals, $2 \mathrm{ml}$ of the supernatant was removed and replenished with an identical volume of fresh buffer. The drug concentrations were determined by a high-performance liquid

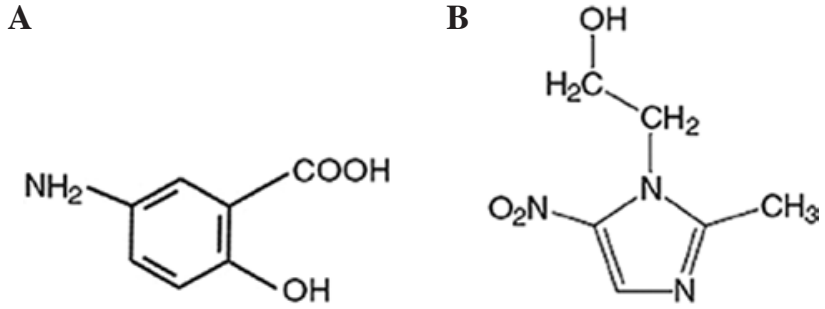

Figure 1. Structural formula of (A) mesalazine and (B) metronidazole.

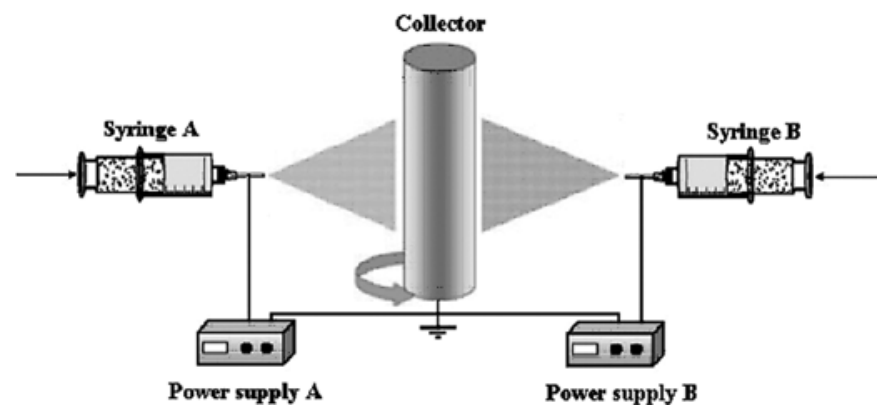

Figure 2. Schematic diagram of the interlaced electrospinning setup.

chromatograph (LabAlliance model 500; USA). Each sample was assayed in triplicate. Then, the accumulative amount of the released drug was calculated as a function of incubation time. The chromatographic conditions (19) were as follows: chromatographic column was a Hypeysil-ODS C18 column. The mobile phase was a 80/20 mixture (v/v) of $\mathrm{pH} 6.5 \mathrm{PBS}$ and methanol, including $0.01 \mathrm{M}$ tetrabutylammonium bromide. The flow rate was $1.0 \mathrm{ml} / \mathrm{min}$, and the detection wavelength was $240 \mathrm{~nm}$.

Stability test. The stability of the fibrous membrane-loaded drugs was tested. Several samples were put in a desiccator at $60^{\circ} \mathrm{C}$ and a humidity dryer at $25^{\circ} \mathrm{C}$ as well as $90 \%$ relative humidity, respectively, and were kept for 12 days. Moreover, lightfastness experiment was also carried out in a light tester (Huanghai Ls3000; Shanghai, China). Illumination was $4,500 \pm 500 \mathrm{Lx}$. The samples were taken out on the 6 th and 12th day, and then their appearance, the amount of loaded drug and rate of release were investigated.

\section{Results and Discussion}

The choice of pharmacological treatment for the prevention of post-operative recurrence in $\mathrm{CD}$ relies on an informed estimate of the risk of recurrence. Due to different pharmacological action, the combination of MEZ and metronidazole has been applied to decrease the risk of recurrence. The two composite fibrous membrane-loaded drugs were produced by interlaced electrospinning (Fig. 2).

Fig. 3 shows the morphological appearance of the fibers loaded with MEZ or metronidazole. The average diameter of the two fiber samples was $\sim 800 \mathrm{~nm}$. The fibrous surface was smooth, and no drug crystals were detected by electronic microscopy, either on the surface of the fibers or outside the fibers, as shown in Fig. 3. This was attributed to the solubility 

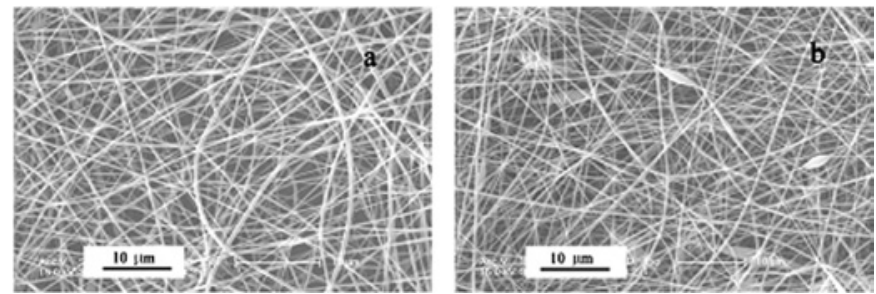

Figure 3. SEM micrographs of fibrous membranes loaded with (a) mesalazine and (b) metronidazole.

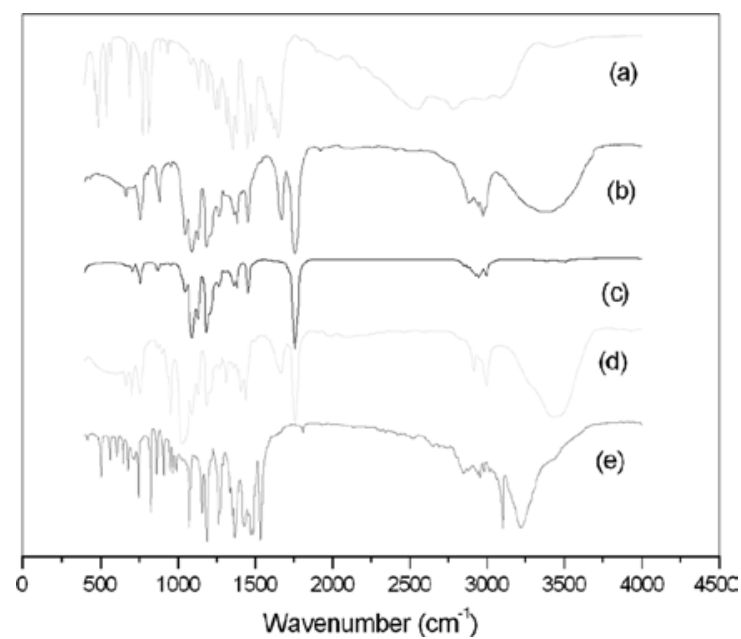

Figure 4. FTIR spectra of (a) mesalazine, (b) mesalazine/PLGA fibers, (c) PLGA fibers, (d) metronidazole/PLGA fibers and (e) metronidazole.

of metronidazole in PLGA/chloform solution, while MEZ was soluble in PLGA/DMF/chloform solution. When the solution jet was rapidly drafted and the solvents evaporated quickly, it was difficult to achieve phase-separation, and the drug was prone to remain inside the fiber where there was enough solvent left. When the fiber became dry, the drug was encapsulated inside.

FTIR spectra of the different samples are presented in Fig. 4. Fig. 4c shows characteristic bands of PLGA $\sim 1,756 \mathrm{~cm}^{-1}$ of $\mathrm{C}=\mathrm{O}, 2,900-3,000 \mathrm{~cm}^{-1}$ of $\mathrm{C}-\mathrm{H}, 1,189$ and $1,131 \mathrm{~cm}^{-1}$ of C-O, which were also observed in the case of two PLGA fibers loaded with the drugs (Fig. 4b and d). In addition, many new peaks appeared in the IR spectra of the loaded drug PLGA fibers, and the characteristic bands of PLGA after incorporating the drugs strengthened, indicating that the drug was mixed with PLGA, and there existed an interaction between PLGA and the drugs.

To determine the effect of the loaded amount on drug release, the in vitro release experiment was carried out by incubating fibrous membranes containing 1 or $2 \%$ wt of MEZ in $0.05 \mathrm{M}$, and $\mathrm{pH} 6.8 \mathrm{PBS}$ solutions at $37^{\circ} \mathrm{C}$. The results are shown in Fig. 5. No burst release of the drugs was observed, indicating the perfect inclusion of the drugs inside the fibers. With an increase in time, the released amount of MEZ from the fibrous membranes increased steadily. The higher the loaded drug amount, the more rapid the MEZ release rate from the fibrous membrane was. For example, the percentages of drug release of the fibrous membrane loaded with 1 and $2 \%$ wt MEZ were 45 and 57\% at 15 days, respectively. It showed

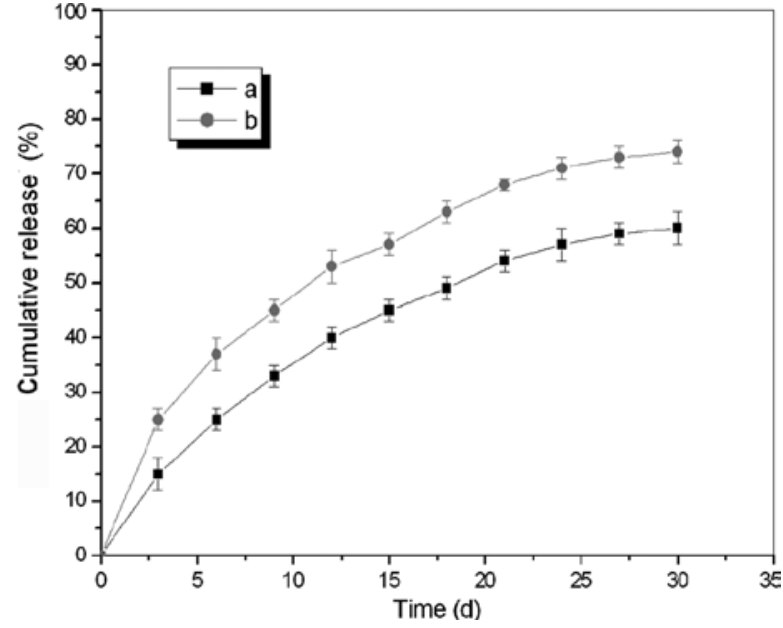

Figure 5. Release curves from the fibrous membrane loaded with (a) $1 \% \mathrm{wt}$ and (b) $2 \%$ wt mesalazine.

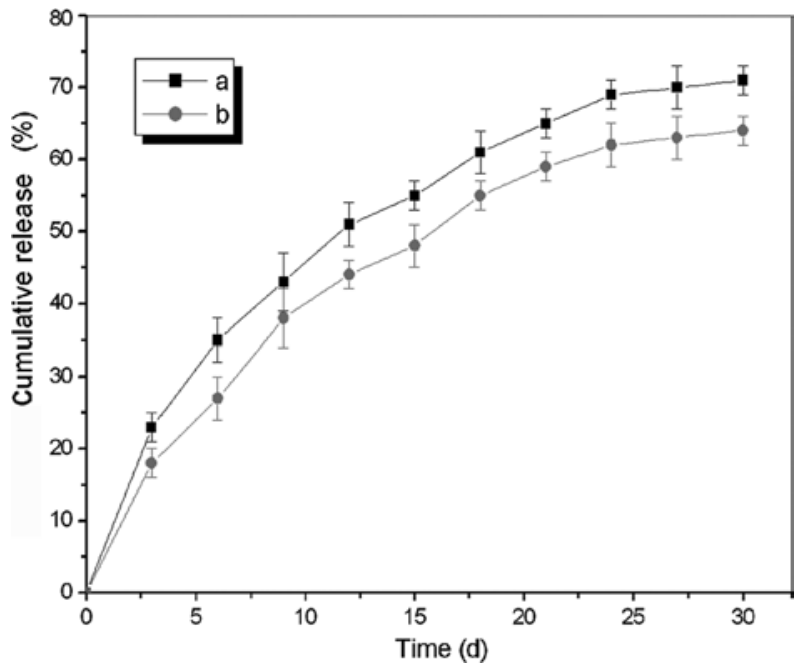

Figure 6. Release curves from the composite fibrous membrane loaded with (a) $2 \% \mathrm{wt}$ mesalazine and (b) $2 \% \mathrm{wt}$ metronidazole.

that the drug release behavior was able to be controlled by adjusting the loaded drug amount. Xu et al (20) also discovered a similar phenomenon when they investigated the in vitro release of BCNU-loaded PEG-PLLA ultra-fine fibers.

Fig. 6a and $b$ illustrates the drug release behaviors of the composite fibrous membranes loaded with 2\% wt MEZ and $2 \%$ wt metronidazole. Both drugs from the fibrous membrane were composed of a firstrapid release phase and a gradual release phase. The MEZ release rate was slightly more reapid than that of metronidazole, although the molecular weight of MEZ (594) was higher than that of metronidazole (177). Mesalazine may create intramolecular or intermolecular hydrogen bonds in solution, which depress the interaction between polymers and MEZ. Due to degradation of the polymer, corrosion also promoted drug release with an increase in time. Moreover, the dosage of MEZ and metronidazole in the fibrous membranes may be controlled easily through adjustment of the volume ratio of the two spin solutions. 
Table I. Result of the stability experiment.

\begin{tabular}{|c|c|c|c|c|c|c|}
\hline \multirow[b]{2}{*}{ Items } & \multicolumn{2}{|c|}{$60^{\circ} \mathrm{C}$} & \multicolumn{2}{|c|}{$\begin{array}{c}\text { Relative } \\
\text { Humidity }=92.5 \%\end{array}$} & \multicolumn{2}{|c|}{ Strong light } \\
\hline & Day 6 & Day 12 & Day 6 & Day 12 & Day 6 & Day 12 \\
\hline Color & - & - & - & - & - & - \\
\hline Increased wt $\%$ & - & - & + & + & - & - \\
\hline \multicolumn{7}{|l|}{ Mesalazine } \\
\hline $\mathrm{C}$ & - & - & - & - & - & - \\
\hline $\mathrm{R}$ & - & + & - & + & - & + \\
\hline \multicolumn{7}{|l|}{ Metronidazole } \\
\hline $\mathrm{C}$ & - & - & - & - & - & - \\
\hline $\mathrm{R}$ & - & + & - & + & - & + \\
\hline
\end{tabular}

-, no significant change; + , a little change. $\mathrm{C}$, content $(\%)$; , release percentage at $5 \mathrm{~h}$ in $\mathrm{pH} 6.8$ phosphate buffer $(\%)$.

Table I shows the effect of different factors on the stability of the fibrous membrane-loaded drugs. The appearance and color of the samples were not changed. Compared to the initial sample, the loaded drug amount only slightly varied, while the drug release percentage increased slightly on the 12th day. This may be due to the degradation of PLGA after testing.

In conclusion, Crohn's disease is one of the most difficult diseases to therapeutically manage in the field of gastroenterology. We firstly prepared a composite fibrous membrane loaded with MEZ and metronidazole by interlaced electrospinning. The two drugs were entrapped in PLGA fibers, respectively, and there was some interaction between the drugs and the polymer. The in vitro release experiment revealed that the interlaced fibrous membrane was able to be used to control release of the two drugs. The loaded drug amount and the drug release rate may be adjusted by changing the volume ratio of the two spin solutions and drug concentrations, respectively. Fibrous membrane-loaded drugs exhibit excellent stability.

\section{References}

1. Fiocchi C: Inflammatory bowel disease: etiology and pathogenesis. Gastroenterology 115: 182-205, 1998.

2. Onderdonk AB, Hermos JA, Dzink JL and Bartlett JG: Protective effect of metronidazole in experimental colitis. Gastroenterology 74: 521-526, 1978.
3. Robinson M: Optimizing therapy for inflammatory bowel disease. Am J Gastroenterol 92: 12-17, 1997.

4. McLeod RS, Wolff BG, Steinhart AH, Carryer PW, O'Rourke K, Andrews DF, Blair JE, Cangemi JR, Cohen Z and Cullen JB: Prophylactic mesalamine treatment decreases postoperative recurrence of Crohn's disease. Gastroenterology 109: 404-413, 1995.

5. Tripathi KD: Essentials of Medical Pharmacology. 5th edition. Jaypee Brothers Medical Publishers (P) Ltd., New Delhi, pp620-621, 2004.

6. Prakash A and Markham A: Oral delayed-release mesalazine: a review of its use in ulcerative colitis and Crohn's disease. Drugs 57: 383-408, 1999.

7. The Merck Index. 11th edition. Merck and Co. Inc., Rahway, NJ, p6181, 2001.

8. Heisterberg L and Branebjerg PE: Blood and milk concentrations of metronidazole in mothers and infants. J Perinatal Medicine 11: 114-118, 1983.

9. Marchioretto MA, Ecclissato C, da Silva CM, Cassiano NM, Calafatti SA, Mendonça S, Ribeiro ML, Bernasconi GC, Degger MF, Piovesan H and Pedrazzoli J Jr: Plasma hydroxymetronidazole/metronidazole ratio in hepatitis $\mathrm{C}$ virus-induced liver disease. Braz J Med Biol Res 38: 437-444, 2005.

10. The Martindale. 35th edition. The Complete Drug Reference. Published Pharmaceutical Press, London, 2006.

11. Hanauer SB and Meyers S: Management of Crohn's disease in adults. Am J Gastroenterol 92: 559-565, 1997.

12. Larrondo L and Manley RSJ: Electrostatic fiber spinning from polymer melts. I. Experimental observations on fiber formation and properties. J Polym Sci Polym Phys Ed 19: 909, 1981.

13. Schreuder-Gibson HL, Gibson P and Senecal K: Protective textile materials based on electrospun nanofibers. J Adv Mater 34: 44-55, 2002.

14. Reneker DH and Chun I: Nanometre diameter fibres of polymer, produced by electrospinning. Nanotechnology 7: 216-223, 1996.

15. Theron SA, Zussman E and Yarin AL: Experimental investigation of the governing parameters in the electrospinning of polymer solutions. Polymer 45: 2017-2030, 2004.

16. Buchko CJ, Chen LC, Shen Y and Martin DC: Processing and microstructural characterization of porous biocompatible protein polymer thin films. Polymer 40: 7397-7404, 1999.

17. Morozov VN, Morozov TY and Kallenbach NR: Atomic force microscopy of structures produced by electrospraying polymer solutions. Int J Mass Spectrom 178: 143-159, 1998.

18. Song XF, Wang C, Zhang DJ: Surface structure and adsorption properties of ultrafine porous carbon fibers. Applied Surface Science 255: 4159-4163, 2009.

19. Shah RS and Shan SA: Spectrophotomatric determinition of masalazine and its dose form. Indian Drugs 31: 34, 1994.

20. Xu X, Chen X, Xu X, Lu T, Wang X, Yang L and Jing X: BCNUloaded PEG-PLLA ultrafine fibers and their in vitro antitumor activity against glioma C6 cells. J Controlled Release 114: 307-316, 2006. 